Review

\title{
CD47/SIRPa pathway mediates cancer immune escape and immunotherapy
}

\author{
Xiao Jia1,2, Bingjun Yan², Xiaoqing Tian², Qian Liu'1,3, Jianhua Jinn ${ }^{1,3}$, Juanjuan Shi², Yongzhong Hou ${ }^{1,2}{ }^{\bowtie}$ \\ 1. Department of Oncology, the Affiliated Wujin Hospital, Jiangsu University, Changzhou, Jiangsu Province, 213017, the People's Republic of China. \\ 2. School of Life Sciences, Jiangsu University, Zhenjiang, Jiangsu Province, 212013, the People's Republic of China. \\ 3. Department of Oncology, the Wujin Clinical College of Xuzhou Medical University, Jiangsu Province, 212017, the People's Republic of China. \\ $\square$ Corresponding author: Yongzhong Hou, houyz@ujs.edu.cn. Xufu Road 308\#, 212000, Zhenjiang, Jiangsu Province, People's Republic of China. Tel: +86 511 \\ 85968935; Fax: +86 51185968934 \\ () The author(s). This is an open access article distributed under the terms of the Creative Commons Attribution License (https://creativecommons.org/licenses/by/4.0/). \\ See http://ivyspring.com/terms for full terms and conditions.
}

Received: 2021.03.23; Accepted: 2021.07.14; Published: 2021.07.25

\begin{abstract}
The adaptive immune checkpoints such as PD-1 (programmed death-1)/PD-L1 (programmed death-ligand 1) play an important role in cancer immunotherapy, whereas increasing evidence suggests that cancer cell evades immune surveillance by innate immune checkpoints such as SIRPa (signal-regulatory protein a)/CD47 (cluster of differentiation 47). In multiple types of cancer cells and solid tumor tissues, highly expressed CD47 protein level has been observed, which is triggered by some transcription factors including NFKB, Myc, and HIF. As a transmembrane protein, the binding of CD47 to SIRPa ligand on phagocytes results in phagocytosis resistance and cancer cell immune escape. In contrast, CD47-SIRPa interaction blockade enhances cancer cell clearance by phagocytes such as macrophages and dendritic cells (DCs) to activate an innate immune response, whereas this process could promote antigen cross-presentation by antigen present cells (APCs) leading to T cell priming, consequently, activates an adaptive antitumor immune response. In this review, we discussed the current SIRPa-CD47 axis-mediated cancer cell immune escape and immunotherapy, which could provide an effective antitumor strategy by the innate and adaptive immune response.
\end{abstract}

Key words: CD47, SIRPa, immune escape, innate immune response, adaptive immune response, cancer immunotherapy

\section{Introduction}

The innate and adaptive immune systems of host play an important role in killing cancer cells and inhibiting tumor progression [1-3], while cancer cell exhibits immune escape by expression of some immune checkpoint proteins such as PD-L1 (programmed death-ligand 1) and CD47 (cluster of differentiation 47) [3, 4]. PD-1(programmed death-1)/ PD-L1 checkpoint functions as "don't find me" signal to the adaptive immune response [5-7], whereas SIRPa (signal-regulatory protein a)-CD47 axis serves as "don't eat me" signal to the innate immune response [7, 8] (Figure 1). The interaction of PD-L1 with surface PD-1 receptor on $\mathrm{T}$ cells leads to inhibition of cancer cell killing [9, 10], whereas the binding of CD47 to surface SIRPa receptor on phagocytes inhibits cancer cell clearance $[7,8,11]$.
CD47 is a widely expressed glycoprotein in normal and cancer cells with five transmembrane domains $[12,13]$, which binds to the extracellular domain of SIRPa on phagocytes leading to inhibition of phagocytosis [7, 12]. The SIRPa/CD47 checkpoint was first identified in 1999 [14, 15], which suppresses phagocytosis of phagocytes and promotes cancer immune escape $[8,16,17]$. The clinical analysis shows that CD47 is highly expressed on multiple types of cancer patients including glioblastoma, ovarian, breast, bladder, colon, and hepatocellular carcinoma, which correlates with low survival [18]. CD47 expression is trigged by multiple transcriptional factors including NFKB, Myc, and HIF, etc [3, 11, 19, 20], while the SIRPa-CD47 axis blockade enhances phagocytosis by macrophages and DCs to activate 
innate immune response resulting in tumor regression $[3,7,11,12,18]$, whereas the phagocytosis by DCs activates DNA-sensing cGAS-STING-INF$\gamma$-mediated adaptive immune response leading to $\mathrm{T}$ cell priming [21-23], suggesting that inhibition of SIRPa-CD47 axis could enhance innate and adaptive antitumor immune response. In this review, we discussed the regulatory mechanism of CD47-mediated cancer immune escape and immunotherapy.

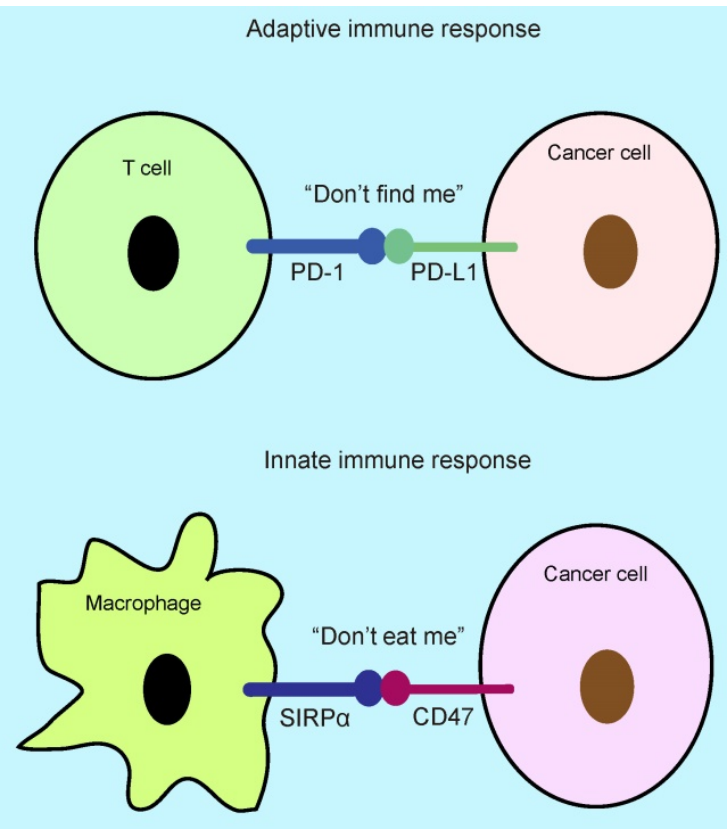

Figure 1. "Don't eat me" and "don't find me" signal. The binding of CD47 to SIRPa on phagocytes inhibits phagocytosis, which functions as "don't eat me" signal, whereas the binding of PD-L1 to PD-1 serves as "don't find me" signal that inhibits T cell killing. APCs: antigen present cells.

\section{SIRPa-CD47 axis protects cancer cell from phagocytosis}

SIRPa is one of the SIRP family members, which was first identified in 1997 [24]. Thibaudeau et al [25] reports that SIRPa is highly expressed on macrophages. After that, CD47 was identified as the first ligand of SIRPa [14, 15]. The binding of SIRPa to CD47 triggers SIRPa phosphorylation of ITIMs (immunoreceptor tyrosine-based inhibitory motifs) resulting in deactivation of myosin IIA, which is a critical step to block phagocytosis [16]. CD47 glycoprotein is highly expressed in multiple types of cancer cells and human tumor tissues [7, 18, 26, 27], which is regulated by Myc oncogene [11]. In this study, Myc directly binds to CD47 promoter and triggers its gene expression. In $\mathrm{T}$ cell acute lymphoblastic leukemia (T-ALL) xenograft tumor model, activation of Myc leads to tumor growth and inhibition of phagocytosis, which is alleviated by Myc inactivation [11]. Interestingly, another report shows that silenced CD47 reduces Myc expression in oral squamous cell carcinoma [27], which suggests that CD47 could increase Myc expression. However, it is unclear whether CD47-Myc-CD47 feedback signal could regulate CD47 expression. In addition to direct regulation of CD47 promoter by Myc binding [11], extracellular stimuli also trigger CD47 gene expression [20, 28]. In response to $\mathrm{TNFa}$, activated NFkB (nuclear factor- $\mathrm{kB}$ ) directly binds to a specific constituent enhancer of CD47 and increases its gene expression in MCF-7 breast cancer cells resulting in tumor growth by inhibiting phagocytosis [28]. Moreover, under hypoxia condition, HIF-1 (hypoxia-inducible factor 1) binds to CD47 promoter and increases its expression resulting in inhibition of phagocytosis in breast cancer cells, which is a strong correlation between CD47 and HIF-1 by clinical analysis from thousands of breast cancer patients [20]. The ChIP-Seq-based analysis shows that nuclear respiratory factor 1 (NRF-1) targets CD47 promoter [29]. Consistent with this, oncogenic activation of ERK signal induces CD47 expression by NRF-1-mediated CD47 gene transcription in melanoma cells leading to inhibition of phagocytosis [30]. In contrast, IDH1 $(\mathrm{R} 132 \mathrm{H})$ mutation in gliomas, negatively regulates CD47 gene transcription, which disrupts the binding of PKM2/ $\beta$-catenin/BRG1 complex to TCF4 transcription factor resulting in inhibition of TCF4-mediated CD47 expression [31]. In addition, Berkovits and Mayr [32] have described the mechanism of how does the new synthesized CD47 to be delivered to the cell surface. This study suggests that CD47 protein is present on the cell surface and intracellular, whereas the long 3'UTR of CD47 is critical for its surface localization. Mechanistically, the binding of $\mathrm{HuR}$ to long 3'UTR recruits SET to develop CD47 mRNA/HuR/SET complex and targets the endoplasmic reticulum (ER) surface, subsequently, the binding of SET to the new synthesized cytoplasmic domains of CD47 recruits RAC1 and forms a CD47/SET/RAC1 complex leading to the plasma membrane translocation of CD47 protein [32]. Moreover, the expression of CD47 protein undergoes transcriptional modification by glutaminyl-peptide cyclotransferase-like (QPCTL), which induces CD47 pyroglutamate formation shortly after biosynthesis [17]. In this study, it shows that the formation of pyroglutamate on CD47 enhances the binding of SIRPa to CD47, consequently, inhibits cancer cell clearance by phagocytes. In addition to present on cell surface, CD47 protein is observed on exosomes [33-35]. Exosomes are extracellular vesicles (30$150 \mathrm{~nm}$ ) with double-layer membrane, which is secreted from cells and effectively enter into other cells [36]. High CD47 levels on the exosomes of breast 
cancer patients may be unfavourable [33, 34], and CD47 on the exosomes inhibits cancer cell clearance by phagocytes in pancreatic cancer [35], while it still unclear the secreted mechanism of CD47 on the exosomes. Taken together, CD47 gene expressions are regulated by multiple transcription factors, which could be transcriptional modification by pyroglutamate formation that enhances the binding of CD47 to SIRPa, consequently, inhibits phagocytosis by phagocytes and promotes cancer cell immune escape. CD47 on the exosomes also decreases antitumor activity by inhibition of phagocytosis. So, the surface CD47 on cancer cells and exosomes should be blocked for cancer immunotherapy (Figure 2).

\section{CD47 blockade enhances the innate and adaptive antitumor immune response}

CD47-SIRPa axis serves as "don't eat me" signal to the innate immune response [7, 8], whereas
SIRPa-CD47 checkpoint blockade promotes phagocytosis by phagocytes such as macrophages and DCs leading to tumor regression by activation of innate immune response [23, 37, 38]. The antitumor activity by CD47 blockade enhances cancer cell clearance by both of phagocytes and T cells [26, 37], and anti-CD47 antibody enhances $\mathrm{CD}^{+} \mathrm{T}$ cells killing but not $\mathrm{CD}^{+} \mathrm{T}$ cell in colon cancer cells [37]. Silenced CD47 in T cells leads to enhanced $\mathrm{T}$ cell killing in irradiated melanoma cells [26]. Vaccination with CD47 knockout tumor cells induces $\mathrm{CD}_{11 \mathrm{c}^{+}}$ SIRPa ${ }^{+} \mathrm{DCs}$ activation and enhances $\mathrm{T}$ cell response in B16F0 melanoma mouse tumor model [39]. As APCs, DCs engulf cancer cells and tumor-derived DNA in DCs activates cGAS (cGAMP), a cytosolic DNA sensor, subsequently, activates the downstream cGAS-cGAMP-STING innate immune response that exhibits antitumor activity [23, 40], whereas highly expressed CD47 inhibits this signaling pathway in

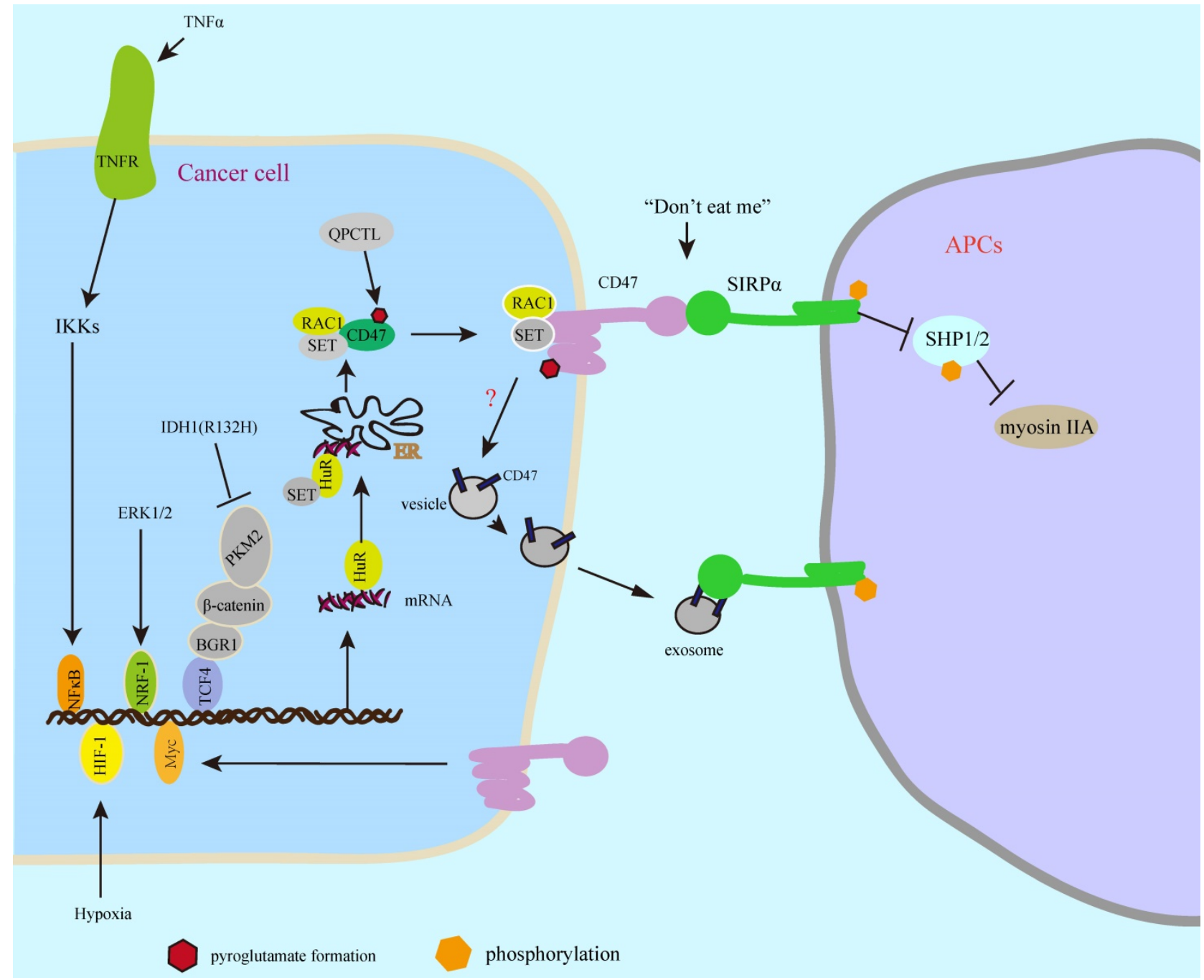

Figure 2. SIRPa-CD47 axis protects cancer cell from phagocytosis. Multiple transcription factors regulate CD47 expression in response to intracellular oncogenic activation pathways or extracellular stimuli. The new synthesized CD47 protein is delivered to the cellular surface by binding to SET/RAC complex proteins, which undergoes pyroglutamate formation by cyclotransferase-like (QPCTL) shortly after biosynthesis leading to increased phagocytosis resistance. In addition, the CD47 protein on the surface of the exosomes inhibits phagocytosis. 
cancer cells leading to tumor immune escape $[7,8]$. CD47-SIRPa blockade by anti-CD47 antibody enhances antigen cross-presentation by DCs and promotes $\mathrm{T}$ cell priming, consequently, $\mathrm{CD}^{+} \mathrm{T}$ cells, but not $\mathrm{CD}^{+} \mathrm{T}$ cells, mediate killing on colon cancer cells. In this process, cytosolic DNA sensor STING (Stimulator of interferon genes) is required for anti-CD47 antibody-mediated tumor regression [23]. In addition, bispecific anti-PD-L1-SIRPa, both of SIRPa/CD47 and PD-1/PD-L1 checkpoints blockade, significantly enhances $\mathrm{CD} 8^{+} \mathrm{T}$ cell killing on colon cancer cells compared to SIRPa/CD47 or PD-1/PD-L1 blockade alone, which is involved in activation of STING-IFN- $\gamma$ pathway in DCs [41]. Combined CD47/SIRPa blockade with temozolomide in glioblastoma enhances phagocytosis and promotes $\mathrm{T}$ cell priming by activation of STING-IFN- $\gamma$ pathway in DCs [38]. In addition to tumor-derived DNA, the tumor mitochondrial DNA (mtDNA) can also trigger the cGAS-cGAMP-STING innate immune response [42]. In this study, it reports that CD47 blockade leads to inhibition of degradation of tumor mtDNA by activation of NADPH oxidase NOX2 in DCs, consequently, activates the mtDNA-cGAS-STINGIFN- $\gamma$ pathway in DCs. These findings suggest that CD47 blockade activates cGAS-cGAMP-STINGmediated innate immune response as well as adaptive immune response by cGAS-STING-IFN- $\gamma$ signalmediated $\mathrm{T}$ cell priming (Figure 3 ).

\section{SIRPa-CD47 checkpoint blockade in cancer immunotherapy}

Increasing evidence suggests that SIRPa-CD47 checkpoint blockade enhances the efficacy of cancer immunotherapy (Table 1). SIRPa-CD47 axis blockade by using anti-CD47 antibody significantly enhances phagocytosis by macrophages and inhibits tumor growth [8, 18, 23, 26, 37, 43]. Furthermore, SIRPa specific monoclonal antibody KWAR23 disrupts SIRPa-CD47 interaction resulting in inhibition of tumor growth by increasing phagocytosis [44].

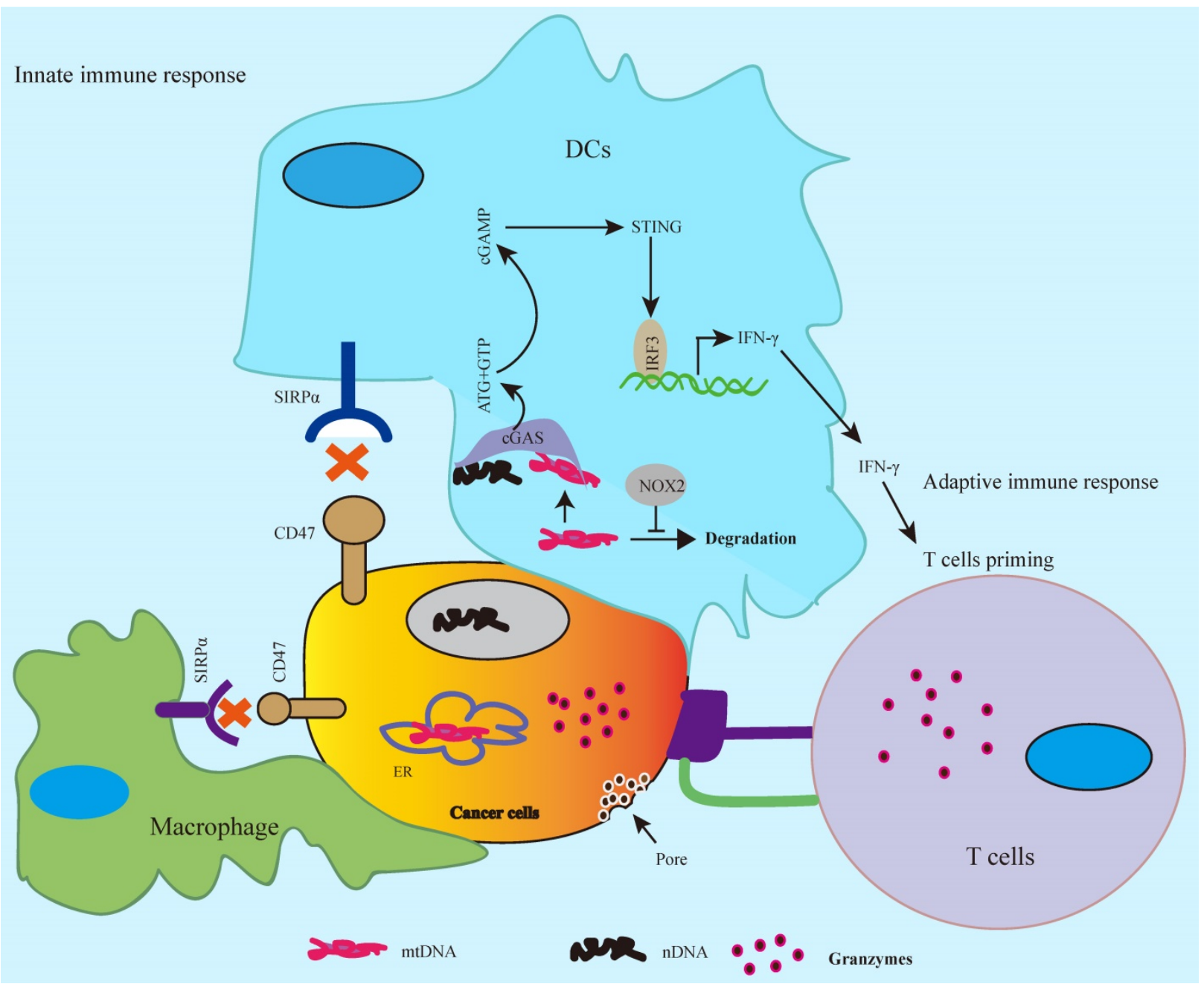

Figure 3. CD47 blockade activates innate and adaptive antitumor immune response. CD47-SIRPa axis blockade activates cGAS-cGAMP-STING-mediated innate immune response by tumor-derived nuclear DNA (nDNA) or mitochondrial DNA (mtDNA) in DCs, whereas the release of IFN-Y via cGAS-cGAMP-STING-INFR signal results in cytotoxic $T$ cell priming and activates adaptive antitumor immune response. 
TTI-621 (SIRPaFc), a recombinant protein for CD47 binding, activates macrophage phagocytosis and inhibits tumor growth $[45,46]$. In addition to block CD47/SIRPa checkpoint alone, combined SIRPa/ CD47 with PD-1/PD-L1 blockade enhances the efficiency of antitumor immunotherapy [41, 47, 48], suggesting that SIRPa-CD47 axis blockade could enhance PD-1/PD-L1 blockade therapy for cancer. The binding of the bispecific anti-PD-L1-SIRPa fusion protein to both of PD-L1 and CD47 on cancer cells significantly enhances antitumor activity in MC-38 colon cell xenograft tumor model [41]. Similarly, anti-CD47 antibody synergizes with PD-L1 blockade for cancer immunotherapy in B16F10 melanoma tumor model [47]. Moreover, combined with chemotherapy or radiotherapy also enhances the efficacy of cancer immunotherapy, which could increase $\mathrm{T}$ cell priming via the release of tumor-derived antigens consequent activation of APCs [21, 22]. Similarly, cotrimoxazole synergizes with anti-CD47 antibody treatment leading to enhanced antitumor activity by both of phagocytosis and cGAS-STING DNA sensing signal [23]. In response to mitoxantrone, anti-CD47 antibody significantly enhances antitumor activity in breast cancer cells [49]. SIRPa-CD47 axis blockade enhances cancer cell clearance by phagocytes, which in turn promotes antigen cross-presentation by APCs resulting in enhanced $\mathrm{T}$ cell priming. Therefore, a rational combination of SIRPa-CD47 axis blockade contributes to cancer immunotherapy (Figure 4, Table $1)$.

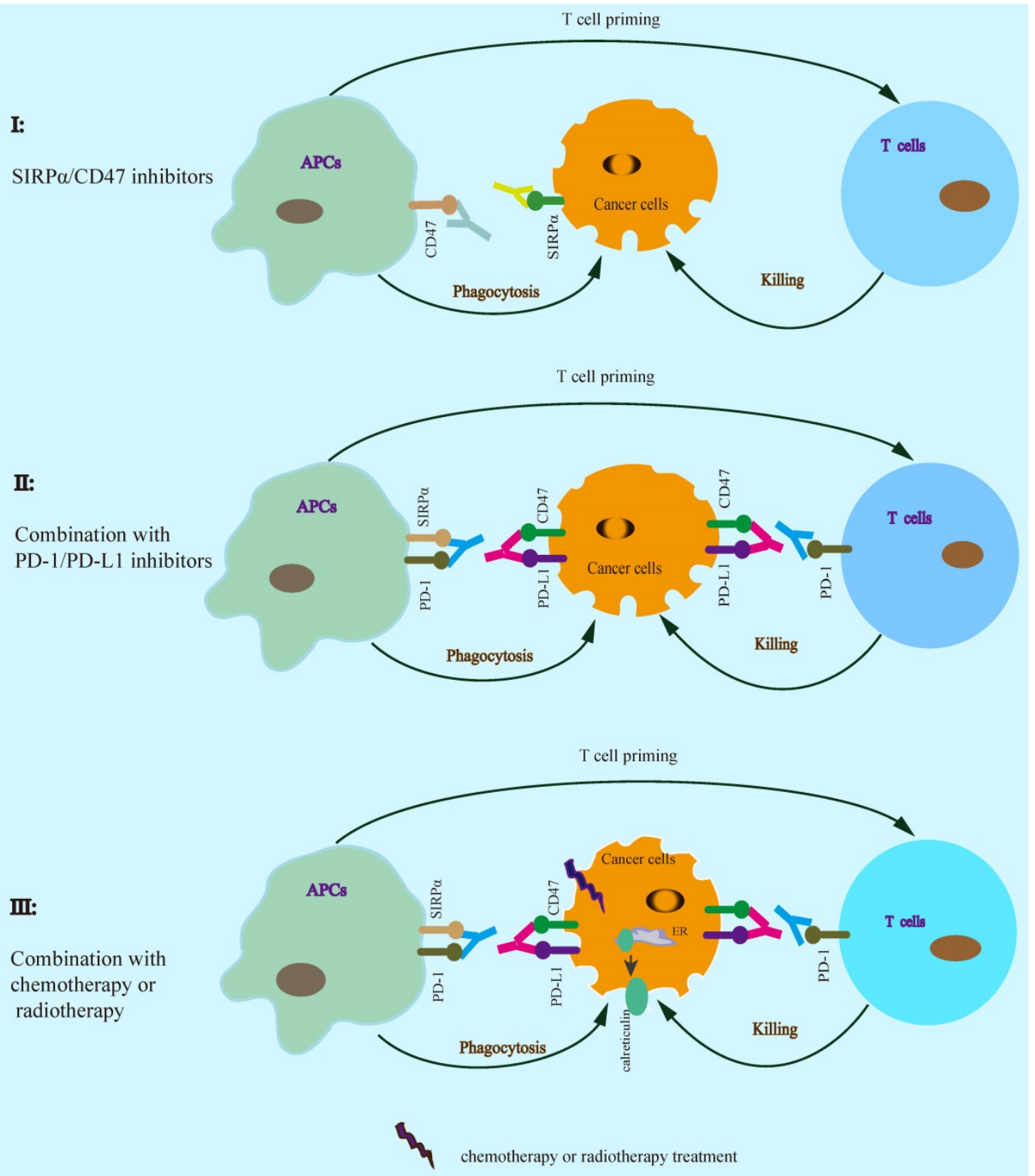

Figure 4. SIRPa-CD47 checkpoint blockade enhances antitumor immunotherapy. A rational combination of SIRPa-CD47 axis blockade contributes to enhancing the efficacy of cancer immunotherapy. 
Table 1. SIRPa/CD47 blockade and antitumor immunotherapy

\begin{tabular}{lll}
\hline Targets & Tumor model & Reference \\
\hline Anti-CD47+BRAF/MEK & Melanoma & 30 \\
inhibitors & & \\
Anti-CD47 & Acute myeloid leukemia (AML) stem cells & 8 \\
Anti-CD47 & Breast cancer & 49 \\
KWAR23 (Anti-SIRPa) & Burkitt's lymphoma & 44 \\
TTI-621 (SIRPaFc) & Lymphoma. AML & 46 \\
Anti-PD-L1-SIRPa & Colon cancer & 41 \\
Anti-CD47+ anti-PD-L1 & Melanoma & 47 \\
Cotrimoxazole+anti-CD47 & Colon, B cell lymphoma & 23 \\
Mitoxantrone+anti-CD47 & Breast cancer & 49 \\
1H9(anti-SIRPa)+anti-PD-L1 & Melanoma & 48 \\
SRF23(anti-CD47) & Burkitt's lymphoma & 43 \\
\hline
\end{tabular}

\section{Conclusion}

Highly expressed CD47 levels are present in multiple types of cancers including solid tumors and hematologic malignancies, which is major regulated by some transcription factors such as NFkB, Myc, HIF-1 and NRF-1. Although CD47 protein on exosomes has been observed, the mechanism of secreted pathway is still unclear. Given that normal and blood red cells are widely expressed CD47 that will limit the efficiency of anti-CD47 antibody therapy, therefore, a specific anti-CD47 antibody for CD47/SIRPa blockade is necessary. Especially, combined CD47/SIRPa with PD-1/PD-L1 checkpoints blockade will be preferred to inhibit cancer cell immune evasion. Since DNA damage stimuli could trigger an adaptive antitumor immune response by DNA-sensing cGAS-STING-INF-y pathway or release of tumor-derived antigens, a rational combined anti-CD47 antibody with chemotherapy or radiotherapy could enhance the efficiency of antitumor immunotherapy.

\section{Abbreviations}

PD-1: programmed death-1; PD-L1: programmed death-ligand 1; SIRPa: signal-regulatory protein a; CD47: cluster of differentiation 47; DCs: dendritic cells; APCs: antigen presenting cells; ITIMs: immunoreceptor tyrosine-based inhibitory motifs; T-ALL: T cell acute lymphoblastic leukemia; NFkB: nuclear factor- Kb; HIF-1: hypoxia-inducible factor 1 ; QPCTL: glutaminyl-peptide cyclotransferase-like; STING: Stimulator of interferon genes.

\section{Acknowledgements}

This work was supported by the National Natural Science Foundation of China (81972618, 81872275); Jiangsu Commission of Health Natural Science Foundation (M2020002), Changzhou Sci \& Tech Program. Grant No. CJ20200004.

\section{Competing Interests}

The authors have declared that no competing interest exists.

\section{References}

1. Gattinoni L, Powell DJ, Jr., Rosenberg SA, Restifo NP. Adoptive immunotherapy for cancer: building on success. Nature reviews Immunology 2006; 6: 383-393.

2. Shanker A, Verdeil G, Buferne M, Inderberg-Suso EM, Puthier D, Joly F et al. CD8 T cell help for innate antitumor immunity. Journal of immunology 2007; 179: 6651-6662.

3. Feng M, Jiang W, Kim BYS, Zhang CC, Fu YX, Weissman IL. Phagocytosis checkpoints as new targets for cancer immunotherapy. Nature reviews Cancer 2019; 19: 568-586.

4. Topalian SL, Drake CG, Pardoll DM. Immune checkpoint blockade: a common denominator approach to cancer therapy. Cancer cell 2015; 27: 450-461.

5. Parsa AT, Waldron JS, Panner A, Crane CA, Parney IF, Barry JJ et al. Loss of tumor suppressor PTEN function increases B7-H1 expression and immunoresistance in glioma. Nature medicine 2007; 13: 84-88.

6. Topalian SL, Drake CG, Pardoll DM. Targeting the PD-1/B7-H1(PD-L1) pathway to activate anti-tumor immunity. Curr Opin Immunol 2012; 24: 207-212.

7. Jaiswal S, Jamieson CH, Pang WW, Park CY, Chao MP, Majeti R et al. CD47 is upregulated on circulating hematopoietic stem cells and leukemia cells to avoid phagocytosis. Cell 2009; 138: 271-285.

8. Majeti R, Chao MP, Alizadeh AA, Pang WW, Jaiswal S, Gibbs KD, Jr. et al. CD47 is an adverse prognostic factor and therapeutic antibody target on human acute myeloid leukemia stem cells. Cell 2009; 138: 286-299.

9. Yokosuka T, Takamatsu M, Kobayashi-Imanishi W, Hashimoto-Tane A, Azuma M, Saito T. Programmed cell death 1 forms negative costimulatory microclusters that directly inhibit $\mathrm{T}$ cell receptor signaling by recruiting phosphatase SHP2. J Exp Med 2012; 209: 1201-1217.

10. Iwai $Y$, Hamanishi J, Chamoto K, Honjo T. Cancer immunotherapies targeting the PD-1 signaling pathway. J Biomed Sci 2017; 24: 26.

11. Casey SC, Tong L, Li Y, Do R, Walz S, Fitzgerald KN et al. MYC regulates the antitumor immune response through CD47 and PD-L1. Science 2016; 352: 227-231.

12. Brown EJ, Frazier WA. Integrin-associated protein (CD47) and its ligands. Trends in cell biology 2001; 11: 130-135.

13. Yanagita T, Murata Y, Tanaka D, Motegi SI, Arai E, Daniwijaya EW et al. Anti-SIRPalpha antibodies as a potential new tool for cancer immunotherapy. JCI Insight 2017; 2: e89140.

14. Seiffert M, Cant C, Chen Z, Rappold I, Brugger W, Kanz L et al. Human signal-regulatory protein is expressed on normal, but not on subsets of leukemic myeloid cells and mediates cellular adhesion involving its counterreceptor CD47. Blood 1999; 94: 3633-3643.

15. Jiang P, Lagenaur CF, Narayanan V. Integrin-associated protein is a ligand for the P84 neural adhesion molecule. The Journal of biological chemistry 1999; 274: 559-562.

16. Tsai RK, Discher DE. Inhibition of "self" engulfment through deactivation of myosin-II at the phagocytic synapse between human cells. J Cell Biol 2008; 180: 989-1003.

17. Logtenberg MEW, Jansen JHM, Raaben M, Toebes M, Franke K, Brandsma AM et al. Glutaminyl cyclase is an enzymatic modifier of the CD47- SIRPalpha axis and a target for cancer immunotherapy. Nature medicine 2019; 25: 612-619.

18. Willingham SB, Volkmer JP, Gentles AJ, Sahoo D, Dalerba P, Mitra SS et al The CD47-signal regulatory protein alpha (SIRPa) interaction is a therapeutic target for human solid tumors. Proceedings of the National Academy of Sciences of the United States of America 2012; 109: 6662-6667.

19. Chang WT, Huang AM. Alpha-Pal/NRF-1 regulates the promoter of the human integrin-associated protein/CD47 gene. The Journal of biological chemistry 2004; 279: 14542-14550.

20. Zhang H, Lu H, Xiang L, Bullen JW, Zhang C, Samanta D et al. HIF-1 regulates CD47 expression in breast cancer cells to promote evasion of phagocytosis and maintenance of cancer stem cells. Proceedings of the National Academy of Sciences of the United States of America 2015; 112: E6215-6223.

21. Vanpouille-Box C, Alard A, Aryankalayil MJ, Sarfraz Y, Diamond JM, Schneider RJ et al. DNA exonuclease Trex1 regulates radiotherapy-induced tumour immunogenicity. Nat Commun 2017; 8: 15618.

22. Gameiro SR, Jammeh ML, Wattenberg MM, Tsang KY, Ferrone S, Hodge JW. Radiation-induced immunogenic modulation of tumor enhances antigen processing and calreticulin exposure, resulting in enhanced T-cell killing. Oncotarget 2014; 5: 403-416.

23. Liu X, Pu Y, Cron K, Deng L, Kline J, Frazier WA et al. CD47 blockade triggers $\mathrm{T}$ cell-mediated destruction of immunogenic tumors. Nature medicine 2015; 21: 1209-1215.

24. Kharitonenkov A, Chen Z, Sures I, Wang H, Schilling J, Ullrich A. A family of proteins that inhibit signalling through tyrosine kinase receptors. Nature 1997; 386: 181-186. 
25. Veillette A, Thibaudeau E, Latour S. High expression of inhibitory receptor SHPS-1 and its association with protein-tyrosine phosphatase SHP-1 in macrophages. The Journal of biological chemistry 1998; 273: 22719-22728.

26. Schwartz AL, Nath PR, Allgauer M, Lessey-Morillon EC, Sipes JM, Ridnour LA et al. Antisense targeting of CD47 enhances human cytotoxic T-cell activity and increases survival of mice bearing B16 melanoma when combined with anti-CTLA4 and tumor irradiation. Cancer Immunol Immunother 2019; 68: 1805-1817.

27. Pai S, Bamodu OA, Lin YK, Lin CS, Chu PY, Chien MH et al. CD47-SIRPalpha Signaling Induces Epithelial-Mesenchymal Transition and Cancer Stemness and Links to a Poor Prognosis in Patients with Oral Squamous Cell Carcinoma. Cells 2019; 8.

28. Betancur PA, Abraham BJ, Yiu YY, Willingham SB, Khameneh F, Zarnegar M et al. A CD47-associated super-enhancer links pro-inflammatory signalling to CD47 upregulation in breast cancer. Nat Commun 2017; 8: 14802.

29. Satoh J, Kawana N, Yamamoto Y. Pathway Analysis of ChIP-Seq-Based NRF1 Target Genes Suggests a Logical Hypothesis of their Involvement in the Pathogenesis of Neurodegenerative Diseases. Gene Regul Syst Bio 2013; 7: 139-152.

30. Liu F, Jiang CC, Yan XG, Tseng HY, Wang CY, Zhang YY et al. BRAF/MEK inhibitors promote CD47 expression that is reversible by ERK inhibition in melanoma. Oncotarget 2017; 8: 69477-69492.

31. Gowda P, Patrick S, Singh A, Sheikh T, Sen E. Mutant Isocitrate Dehydrogenase 1 Disrupts PKM2-beta-Catenin-BRG1 Transcriptional Network-Driven CD47 Expression. Molecular and cellular biology 2018; 38: e00001-18.

32. Berkovits BD, Mayr C. Alternative $3^{\prime}$ UTRs act as scaffolds to regulate membrane protein localization. Nature 2015; 522: 363-367.

33. Kibria G, Ramos EK, Lee KE, Bedoyan S, Huang S, Samaeekia R et al. A rapid, automated surface protein profiling of single circulating exosomes in human blood. Scientific reports 2016; 6: 36502 .

34. Wang M, Ji S, Shao G, Zhang J, Zhao K, Wang Z et al. Effect of exosome biomarkers for diagnosis and prognosis of breast cancer patients. Clin Transl Oncol 2018; 20: 906-911.

35. Kamerkar S, LeBleu VS, Sugimoto H, Yang S, Ruivo CF, Melo SA et al. Exosomes facilitate therapeutic targeting of oncogenic KRAS in pancreatic cancer. Nature 2017; 546: 498-503.

36. Colombo M, Raposo G, Thery C. Biogenesis, secretion, and intercellular interactions of exosomes and other extracellular vesicles. Annu Rev Cell Dev Biol 2014; 30: 255-289.

37. Tseng D, Volkmer JP, Willingham SB, Contreras-Trujillo H, Fathman JW, Fernhoff NB et al. Anti-CD47 antibody-mediated phagocytosis of cancer by macrophages primes an effective antitumor T-cell response. Proceedings of the National Academy of Sciences of the United States of America 2013; 110: 11103-11108.

38. von Roemeling CA, Wang Y, Qie Y, Yuan H, Zhao H, Liu X et al. Therapeutic modulation of phagocytosis in glioblastoma can activate both innate and adaptive antitumour immunity. Nat Commun 2020; 11: 1508.

39. Li Y, Zhang M, Wang X, Liu W, Wang H, Yang YG. Vaccination with CD47 deficient tumor cells elicits an antitumor immune response in mice. Nat Commun 2020; 11: 581.

40. Chen Q, Sun L, Chen ZJ. Regulation and function of the cGAS-STING pathway of cytosolic DNA sensing. Nat Immunol 2016; 17: 1142-1149.

41. Liu X, Liu L, Ren Z, Yang K, Xu H, Luan Y et al. Dual Targeting of Innate and Adaptive Checkpoints on Tumor Cells Limits Immune Evasion. Cell Rep 2018; 24: 2101-2111.

42. Xu MM, Pu Y, Han D, Shi Y, Cao X, Liang H et al. Dendritic Cells but Not Macrophages Sense Tumor Mitochondrial DNA for Cross-priming through Signal Regulatory Protein alpha Signaling. Immunity 2017; 47: 363-373 e365.

43. Peluso MO, Adam A, Armet CM, Zhang L, O'Connor RW, Lee BH et al. The Fully human anti-CD47 antibody SRF231 exerts dual-mechanism antitumor activity via engagement of the activating receptor CD32a. J Immunother Cancer 2020; 8: e000413.

44. Ring NG, Herndler-Brandstetter D, Weiskopf K, Shan L, Volkmer JP, George $\mathrm{BM}$ et al. Anti-SIRPalpha antibody immunotherapy enhances neutrophil and macrophage antitumor activity. Proc Natl Acad Sci U S A 2017; 114: E10578-E10585.

45. Petrova PS, Viller NN, Wong M, Pang X, Lin GH, Dodge K et al. TTI-621 (SIRPalphaFc): A CD47-Blocking Innate Immune Checkpoint Inhibitor with Broad Antitumor Activity and Minimal Erythrocyte Binding. Clin Cancer Res 2017; 23: 1068-1079.

46. Lin GHY, Chai V, Lee $\mathrm{V}$, Dodge $\mathrm{K}$, Truong $\mathrm{T}$, Wong $\mathrm{M}$ et al. TTI-621 (SIRPalphaFc), a CD47-blocking cancer immunotherapeutic, triggers phagocytosis of lymphoma cells by multiple polarized macrophage subsets. PLoS One 2017; 12: e0187262.

47. Sockolosky JT, Dougan M, Ingram JR, Ho CC, Kauke MJ, Almo SC et al. Durable antitumor responses to CD47 blockade require adaptive immune stimulation. Proceedings of the National Academy of Sciences of the United States of America 2016; 113: E2646-2654.

48. Liu J, Xavy S, Mihardja S, Chen S, Sompalli K, Feng D et al. Targeting macrophage checkpoint inhibitor SIRPalpha for anticancer therapy. JCI Insight 2020; 5: e134728.

49. Iribarren K, Buque A, Mondragon L, Xie W, Levesque S, Pol J et al. Anticancer effects of anti-CD47 immunotherapy in vivo. Oncoimmunology 2019; 8: 1550619 . 